\section{Asthma + Rhinitis = erhöhtes Notfall-Risiko}

\section{Schon seit längerem wird vermutet, dass Asthmapatienten mit gleichzeitiger allergischer Rhinitis einen schwereren Krankheitsver- lauf zeigen als solche ohne diese Begleiterkrankung. Das bestätigt jetzt auch die Post-hoc-Analyse einer randomisierten Studie.}

H erangezogen wurden die Daten der IMPACT-Studie (IMProving Asthma Control Trial), einer über 52 Wochen durchgeführten doppelblinden Multicenterstudie. Alle 1.490 Teilnehmer hatten vor der Studie an chronischem Asthma gelitten, das durch alleinige Fluticason-Therapie nicht ausreichend zu stabilisieren war. Randomisiert wurden zusätzlich zu zweimal täglich $100 \mu \mathrm{g}$ Fluticason entweder einmal täglich $10 \mathrm{mg}$ Montelukast oder zweimal täglich $50 \mu \mathrm{g}$ Salmeterol verabreicht. Bei der nachträglichen Analyse wurden Patienten mit alleinigem Asthma und solche, die zusätzlich eigenanamnestisch eine allergische Rhinitis angaben, miteinander verglichen.

Eine begleitende Rhinitis lag bei 60\% der Asthmapatienten vor. Diese Patienten waren im Durchschnitt jünger als die Vergleichspatienten und hatten ein weniger schweres Asthma. Umso überraschender ergab die univariate Analyse für Patienten mit einer Begleitrhinitis ein signifikant häufigeres Aufsuchen von Notfallambulanzen sowie eine signifikant höhere Zahl von Asthmaexazerbationen im Vergleich zu Patienten nur mit Asthma $(3,6$ vs. $1,7 \%, p=0,029 ; 21,3$ vs. $17,1 \%, p=0,046)$. Die multivariate Analyse, u.a. unter Berücksichtigung von

\title{
Feinstaub belastet Ältere
}

\section{Über den Zusammenhang zwischen der Exposition gegenüber Staub- partikeln unter 2,5 $\mu \mathrm{m}\left(\mathrm{PM}_{2,5}\right)$ und gesundheitlichen Risiken liegen bislang nur wenig Daten vor. Eine Studie wertete als Maß für eine Beeinträchtigung die stationären Aufnahmen älterer Patienten bei erhöhten $\mathrm{PM}_{2,5}$-Konzentrationen}

\begin{abstract}
A 1s Feinstaub gelten Partikel in der Luft mit einer aerodynamischen Partikelgröße von $10 \mu \mathrm{m}\left(\mathrm{PM}_{10}\right)$ oder darunter. Es wird aber vermutet, dass kleinere Partikel noch tiefer in die Atemwege eindringen und dort schädigend wirken. Aus diesem Grund hat die US-Umweltbehörde die Messung von $\mathrm{PM}_{2,5}$ als zusätzlichen Standard vorgeschrieben.

In einer amerikanischen Untersuchung wurde jetzt über einen Zeitraum von drei Jahren die Zahl der stationären Aufnahmen in 204 städtischen Regionen analysiert. Von 11,5 Millionen stationären Aufnahmen von Patienten im Alter von über 65 Jahren wurden solche wegen kardiovaskulärer und pneumologischer Erkrankungen ausgewertet, bei denen die Patienten im Umkreis von 5,9 Meilen um eine $\mathrm{PM}_{2,5}$-Messstelle wohnten.
\end{abstract}

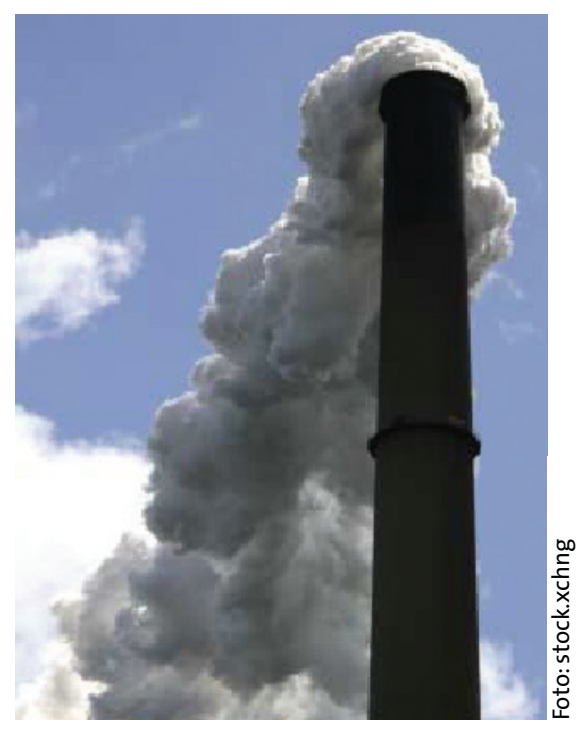

Die Feinstaubbelastung schlägt sich umgehend auf die Gesundheit älterer Menschen nieder.
Behandlungsgruppen, Alter und Asthmaschwere, bestätigte diesen Zusammenhang (Odds Ratio [OR] für Notfälle 2,35; OR für Asthmaattacken 1,35). Die Zahl der ungeplanten Arztbesuche oder Hospitalisierungen war bei zusätzlicher Rhinitis dagegen nicht erhöht.

Fazit: Asthmapatienten mit einer begleitenden allergischen Rhinitis hatten im Vergleich zu solchen ohne Rhintis trotz geringerer Asthmaschwere in dieser Studie ein erhöhtes Risiko für Asthmaattacken und vor allem für das Aufsuchen von Notfallambulanzen. Die Ergebnisse dieser Post-hoc-Analyse müssen aber noch in prospektiven Studien bestätigt werden.

Bousquet J et al. Increased risk of asthma attacks and emergency visits among asthma patients with allergic rhinitis: a subgroup analysis of the improving asthma control trial. Clin Exp Allergy 2005; 35: 723-7

An den Tagen, an denen die $\mathrm{PM}_{2,5}$ zunahm, wurden signifikant mehr Patienten stationär aufgenommen. Dies galt am Tag der erhöhten Partikelkonzentration für zerebrovaskuläre Erkrankungen, Herzinsuffizienz, Herzrhythmusstörungen (hier nur Patienten über 75 Jahren) und chronisch obstruktive Lungenerkrankungen. Mit einer Verzögerung von zwei Tagen mussten auch vermehrt Patienten mit ischämischen Herzerkrankungen und Atemwegsinfekten aufgenommen werden. Am stärksten nahmen die Einweisungen wegen Herzinsuffizienz $\mathrm{zu}\left(+1,28 \%\right.$ je $10 \mu \mathrm{g} / \mathrm{m}^{3}$ Zunahme der $\mathrm{PM}_{2,5}$-Konzentration). Bei den übrigen Erkrankungen lag die Zunahme jeweils unter einem Prozent.

Fazit: Bereits eine kurzfristige Zunahme der $\mathrm{PM}_{2,5}$-Konzentration in der Luft führte in dieser epidemiologischen Untersuchung rasch zu signifikant mehr Krankenhausaufnahmen älterer Patienten mit Herz-Kreislauf-Problemen und Atemwegserkrankungen.

Dominici $\mathrm{F}$ et al. Fine particulate air pollution and hospital admission for cardiovascular and respiratory diseases. JAMA 2006; 295: 1127-34 